\title{
Magnitude estimation of
}

\section{multidimensional stimuli*}

\author{
JOEL FELDMAN and JOHN C. BAIRD \\ Dartmouth College, Hanover, New Hampshire 03755
}

The purpose of this study was to determine the feasibility of magnitude estimation in the multidimensional case. Ss made magnitude estimates of brightness (B) only, loudness (L) only, and brightness and loudness (BL) presented together. The exponent for loudness was the largest, the exponent for brightness was the smallest, and the exponent in the BL condition was equal to the arithmetic mean of the $B$ and $L$ exponents. Two mathematical models were developed to predict the findings for the BL condition on the basis of data obtained for the component attributes. Overall results suggest that brightness and loudness are not perceptually integrated.

One of the more fundamental areas in perception concerns models for the judgment of multidimensional stimuli. Alternative approaches to modeling are found in multidimensional scaling (Shepard, 1964), information theory (Garner (1962), and space perception (Baird, 1970a), but none of these fields seem relevant to the full range of phenomena that must be understood. The complexity of the problem is indicated by the following considerations. On the stimulus side, we must attempt to understand the effects of different dimensions (attributes) as well as the effects of various combinations of intensities taken from multiple dimensions (e.g., orthogonal vs redundant combinations; Garner, 1970). On the response side, we must try to understand how Ss integrate values from multiple dimensions, and how this "integrality" factor can be represented mathematically (Garner, 1970; Lockhead, 1966). Finally, there remains the task of constructing mathematical models for the multidimensional case (Anderson, 1970; Ericksen \& Hake, 1955).

However, if one believes that a general theory of psychophysics is possible, the particular method employed in the multidimensional case is relevant only insofar as it enhances the quantitative representation of results (Baird, 1970a, b, c). The method of magnitude estimation allows for adequate quantification, but, so far as we know, it has never been used to investigate the judgment of combined intensities from multiple dimensions (see, however, the related work by Curtis, Attneave, \&

* This research was supported in part by a mant from the U.S. Public Health Service (4 R01 MH 14379-02) We appreciate the expert advice given by Virgil Graf and W. L. Gulick on the arrangement of the optical and sound equipment used in this study. Requests for reprints should be sent to John C. Baird, Department of Psychology Dartmouth College, Hanover, New Hampshire 03755 .
Harrington, 1968). The advantages of the method of magnitude estimation are that (1) it yields results which are usually fit by a power function, (2) the exponent of the power function is a measure of judgment sensitivity, and (3) models for the multidimensional case can be based on equations obtained for the unidimensional components. The only remaining question is whether or not Ss can make consistent magnitude estimates of intensity based upon multidimensional inputs. The findings reported in this paper indicate that Ss are able to perform such judgment and that relevant models can therefore be constructed.

\section{EXPERIMENT 1}

The purpose of Experiment 1 was to obtain magnitude estimates of multidimensional stimuli and to compare these with estimates of the unidimensional components. The power function is the basic equation used to evaluate results, but we conceive of the details of the function in a somewhat different manner than most investigators. In our formulation, the exponent consists of a ratio between a perceptual and cognitive factor (Baird, 1970c). However, for the purpose of this experiment, it can be assumed that the cognitive factor is constant, and consider the traditional form of the equation:

$$
\mathbf{R}=\alpha \mathbf{S}^{\mathbf{n}},
$$

where $\mathbf{n}$ varies directly as a function of the perceptual factor (Baird, 1970c; Baird, Romer, \& Stein, 1970). No attempt has been made yet to extend this assumption to multidimensional stimuli, since the relevant empirical data do not exist for magnitude estimation, and the theoretical derivation has been unsettled. The present study represents preliminary work toward extension of the theory to the multidimensional case.

Let $n_{1}$ and $n_{2}$ be the exponents for the unidimensional attributes $A_{1}$ and $A_{2}$, and $n_{1,2}$ be the exponent of the multidimensional attribute obtained by combining $A_{1}$ and $A_{2}$. Then, we assume a $\beta$ exists such that

$$
\beta\left(\mathbf{n}_{1}+\mathbf{n}_{2}\right)=\mathrm{n}_{1,2}
$$

or,

$$
\beta=\mathrm{n}_{1,2} /\left(\mathrm{n}_{1}+\mathrm{n}_{2}\right)
$$

Three major hypotheses are possible concerning the value of $\beta$ : (1) The component exponents $n_{1}$ and $n_{2}$ are additive in such a way that $n_{1,2}$ is greater or less than both $n_{1}$ and $n_{2}$. That is, if $n_{1,2}$ is greater than both $n_{1}$ and $\mathbf{n}_{2}$, it follows that

$$
\beta>\left[\mathrm{n}_{1} /\left(\mathrm{n}_{1}+\mathrm{n}_{2}\right)\right]
$$

and

$$
\beta>\left[\mathrm{n}_{2} /\left(\mathrm{n}_{1}+\mathrm{n}_{2}\right)\right] .
$$

If $n_{1,2}$ is less than both $n_{1}$ and $n_{2}$, it follows that

$$
\beta<\left[\mathbf{n}_{1} /\left(\mathbf{n}_{1}+\mathbf{n}_{2}\right)\right]
$$

and

$$
\beta<\left[\mathbf{n}_{2} /\left(\mathbf{n}_{1}+\mathbf{n}_{2}\right)\right]
$$

(2) The exponent $n_{1,2}$ is equal to one of the component exponents, and consequently, either

$$
\beta=\mathbf{n}_{1} /\left(\mathbf{n}_{1}+\mathbf{n}_{2}\right)
$$

or

$$
\beta=\mathbf{n}_{2} /\left(\mathbf{n}_{1}+\mathbf{n}_{\mathbf{2}}\right) .
$$

(3) The exponent $n_{1,2}$ lies somewhere between $n_{1}$ and $n_{2}$. If we assume $\mathbf{n}_{1}>\mathbf{n}_{2}$, then

$$
\left[n_{1} /\left(n_{1}+n_{2}\right)\right]>\beta>\left[n_{2} /\left(n_{1}+n_{2}\right)\right] \text {. }
$$

Empirical data on information transmission suggest that Eqs. 4, 5, and 6 are all tenable under different experimental conditions (Garner, 1962 , 1970). Although no specific decision between these three equations was necessary at the outset, we were led to favor Eqs. 5 and 6 because our particular component attributes (brightness and loudness) seemed to lack perceptual "integrality." Actually, the empirically determined value of $\beta$ can serve as a quantitative measure of "integrality."

Empirical tests of Eqs. 4, 5, and 6 were conducted with sound and luminance intensities which were combined to produce multidimensional stimuli. Exponents 
$\left(n_{1}, n_{2}\right.$, and $\left.n_{1,2}\right)$ were obtained for the three cases, allowing a direct calculation of $\beta$.

\section{Method}

Subjects. The 30 male Ss were students in an introductory psychology course at Dartmouth College and none had any previous experience with the conditions of the experiment. Each stated that he had normal vision and hearing, and those who wore glasses did so during the experiment.

Apparatus. Two rooms were used, one for the $S$ and the other for $E$ and the control equipment. The S's room was approximately $9 \times 8 \mathrm{ft}$ with an 8-ft ceiling. The rooms were acoustically insulated and connected by a $10 \times 10$ in. double-paned window in the common wall. The window was filled by a dull black mask with a 3-in.-diam hole in the center, over which a translucent screen was placed. Architectural drawing paper was placed over the window, and black cardboard with a 3-in.-diam hole in the middle was placed over the paper. The $\mathrm{S}$ sat $3 \mathrm{ft}$ from the light, with his eyes directly in line with the target.

The luminances were produced by passing light from a GE $500-W$ projection bulb through a heat filter and then through a series of condensing lenses, resulting in a 3-in.-diam beam. The intensity of the beam was altered at this point by Kodak neutral filters. The visual target was produced by projecting the beam of light through the window from the control room onto the screen. The beam then passed through another lens that focused it on the rear of the visual target. When viewed from S's room, only a 3-in. circle of diffuse white light was seen. The light source was placed just far enough from the window so that the diameter of the beam was equal to the target diameter. The entire optical system was enclosed in a tunnel of black felt to control extraneous light. The neutral density filters were placed on the ends of levers which were depressed to produce the desired intensity. Ten luminance levels were used: $6.4,16$, $45,78.6,140.4,239.8,421.2,680.4$, $1,112.4$, and $1,808.9 \mathrm{fL}$, as measured with a MacBeth illuminometer. For convenience, each stimulus was assigned a ratio value (a normalized ratio) as compared to the dimmest luminance which was assigned a value of 1.0. This procedure creates ratios of $1.0,2.5,7.03,12.28,21.93,37.47$, 65.81, 106.3, 173.8, and 282.6. Presentation of the visual stimulus was controlled by a solenoid-operated shutter.

The sound stimuli were produced by a Hewlett-Packard Model $200 \mathrm{AB}$ audio oscillator. The audio signal $(1,000 \sim$ tone) was fed from the oscillator to one of 10 preset potentiometers, each adjusted to produce one of the 10 intensities. The signal was then fed to a Dynaco Mark III power amplifier, which provided an output to an Altec Lansing Model 406B unbaffled 10-in. speaker. The center of the speaker was 8 in. above the center of the visual target. Presentation of the audio stimuli was controlled by a relay placed in series with the amplifier output. The relative intensities of the sound stimuli coincided with those of the luminance values. To calculate a set of sound intensities with the same ratios as the luminance values, a $60-\mathrm{dB}$ re $.0002 \mathrm{dyne} / \mathrm{cm}^{2}$ level was selected as the bottom of the scale and given a normalized value of 1.0 . The appropriate decibel levels $-60,68,77$, $82,87,91.5,96,100.5,105$, and $109 \mathrm{~dB}-$ were then calculated and produced. Since decibels are logarithmic units, the normalized antilog of these values are in the same ratios of intensities as the luminance values measured in foot-lamberts. The background noise level of $S$ 's room was $30 \mathrm{~dB}$ re $.0002 \mathrm{dynes} / \mathrm{cm}^{2}$. The $S$ 's verbal responses were transmitted by a lavalier microphone hanging around his neck.

Procedure. During an experimental session, $\mathrm{S}$ was given three conditions: brightness (B) only, loudness (L) only, and brightness and loudness (BL) simultaneously. There were $10 \mathrm{BL}$ combinations, each consisting of a pair of luminance and sound stimuli having the same normalized ratio value; that is, the dimmest light was paired with the softest sound, the most intense light with the loudest sound, etc.

The method of magnitude estimation was used. A condition consisted of two blocks of 10 stimulus presentations each. All 10 stimuli of the condition were presented once in a block in a randomized order. In each condition, the stimulus which had a normalized value of 1.0 was presented before each block of trials as a standard. Every time the standard was presented, it was identified by the $E$ and assigned a value of 1.0 . As the different intensities were presented, the $S$ responded with a number describing how many times more or less intense the comparison was than the standard. In the BL condition, the $S$ was told, "You will be presented with a brightness stimulus and a loudness stimulus simultaneously. Before we begin, you will be presented with a standard brightness-loudness pair whose total intensity, when taken together as one unit, has a value of 1.0. As each of the other brightness-loudness pairs are presented, give me the number describing how many times greater or less the total intensity of that brightness-loudness stimulus, taken together as one unit, is than the total intensity of the standard brightness-loudness pair. For example, if the total intensity of the brightness-loudness pair when taken together as one unit seems five times as intense as the total intensity of the standard, then you would say 'five.' " Each stimulus or stimulus compound was presented for $5 \mathrm{sec}$ with a 5 -sec intertrial interval.

The Ss were divided into six equal groups, each of which received one of the six possible presentation orders of the three conditions, allowing analysis of first judgments independent of exposure to another condition. Each $\mathrm{S}$ was adapted in silence and darkness $10 \mathrm{~min}$ previous to testing.

Results and Discussion

Arithmetic means were computed for within-S estimates of each stimulus magnitude, and geometric means were computed to summarize the arithmetic means.

The empirical validity of the power function was tested by taking the logarithm of both sides of Eq. 1 and performing a least-squares solution to find the slope and $y$-intercept of the linear function. In this case, the slope corresponds to the exponent $(n)$, and

Table 1

Exponents (n), y-Intercepts $(\alpha)$, and Coefficients of Determination $\left(r^{2}\right)$ for Loudness, Brightness, and Brightness-Loudness. Data based on least-squares solutions for Equation 1

Order of Presentation

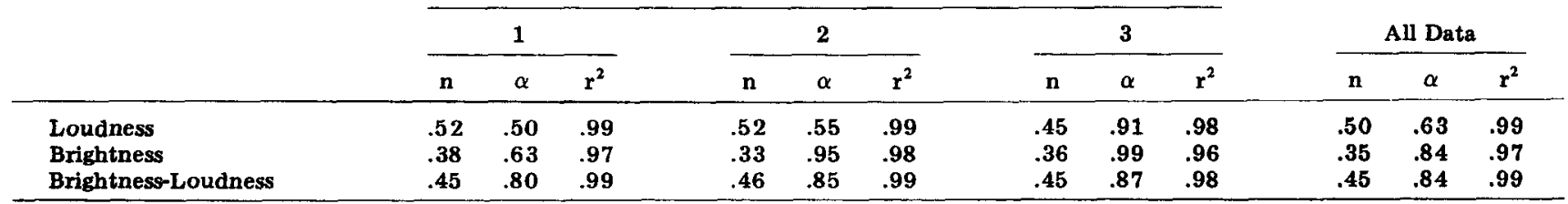




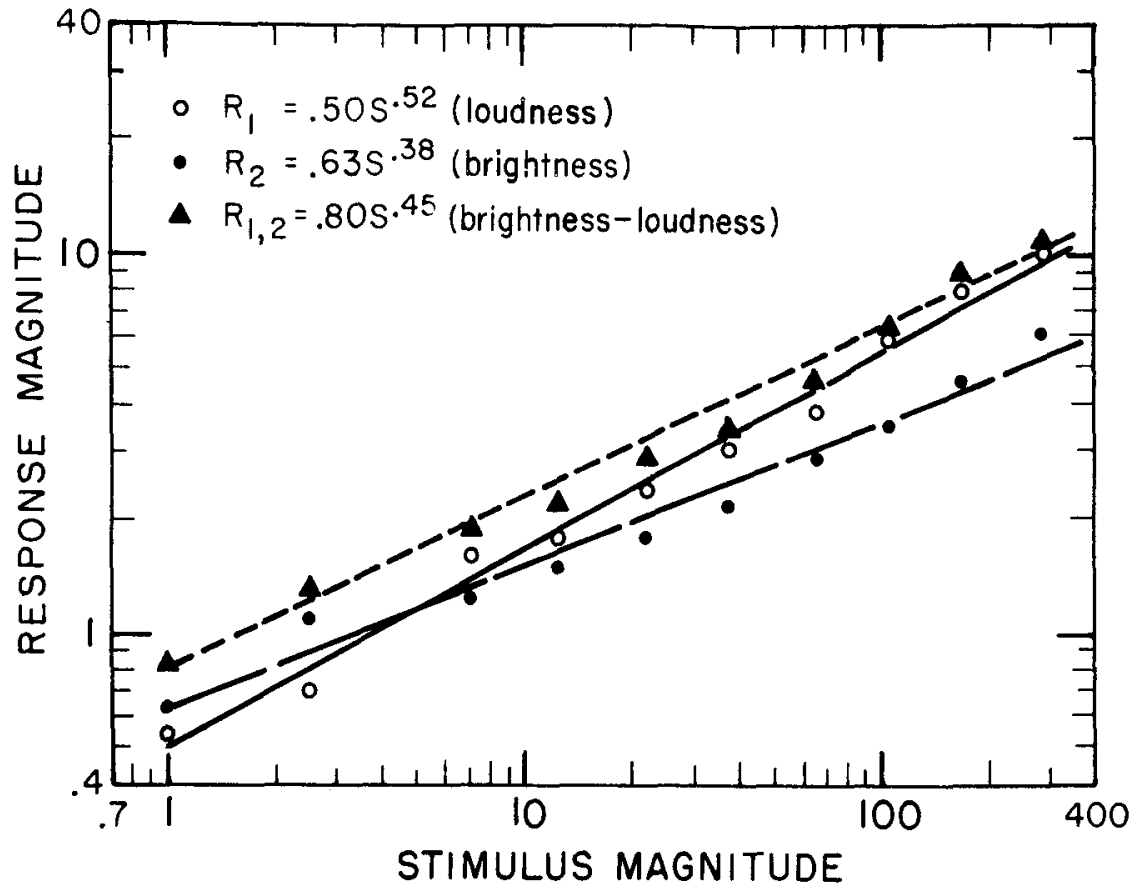

Fig. 1. Response magnitude as a function of stimulus magnitude in logarithmic coordinates. Each point represents the geometric mean of magnitude estimates obtained from 10 Ss in Experiment 1. Different Ss estimated intensities for the attributes of brightness, loudness, and brightness-loudness combinations. The equations correspond to the power functions ( $\mathrm{Eq} .1$ ) obtained by finding the best-fitting straight lines to the logarithmic forms shown on the graphs. For more details, see the text.

the antilog of the $y$-initercept is the multiplicative constant $(\alpha)$ in Eq. 1 . The stimulus levels evaluated were the normalized ratios, which were identical for the $B, L$, and $B L$ conditions. The compatibility of the BL condition with the component attributes was based upon the assumption that correlated stimulus values could be treated as a unitary scale. Least-squares solutions were calculated separately for each attribute condition for first, second, and third orders of presentation in addition to treatment of the data from all $30 \mathrm{Ss}$ regardless of order.

Table 1 presents the exponents (n), multiplicative constants $(\alpha)$ and coefficients of determination $\left(\mathrm{r}^{2}\right)$ for each condition. The high percentages of variance accounted for indicate that good linear fits were obtained. The main concern was with the exponents, the relative order of magnitude of which was generally the same for the three attributes regardless of presentation order. Loudness $\left(\mathrm{n}_{1}\right)$ yielded the highest exponent, brightness $\left(n_{2}\right)$ the lowest, and the BL condition $\left(n_{1,2}\right)$ yielded a value in between that found for the component attributes. The exception to this occurred when an attribute was presented last. Here the exponent for loudness dropped from its value for earlier orders and equaled the exponent for the BL condition. Since an interaction effect was indicated, the analyses to be reported next were carried out only for first estimates.

Figure 1 presents the geometric means of first estimates as a function of stimulus magnitude in logarithmic coordinates. The differences in slopes are clear despite differences in the $y$-intercepts.

In considering the agreement between the results and the predictions, it can be seen from Table 1 that the actual ordering of the exponents was $n_{1}>n_{1,2}>n_{2}$, where $\mathrm{n}_{1}=$ loudness, $\mathrm{n}_{2}=$ brightness, and $n_{1,2}=$ brightness-loudness. The value of $\beta$ is .50 , while $n_{1} /\left(n_{1}+n_{2}\right)=.58$ and $n_{2} /\left(n_{1}+n_{2}\right)=.42$. Since $\beta$ falls between .58 and .42 , Eq. 6 is supported while Eqs. 4 and 5 are not.

It is of interest to find out if these results occur for individuals as well as for groups. Unfortunately, the interaction between order of attribute presentation and the magnitude of the exponent precludes a definitive statement - on this question. Nevertheless, some general indication of the relations for individual Ss can be obtained. Exponents were calculated for each $S$ for each condition, regardless of order of presentation. The individual $\beta$ s were roughly normally distributed, with a preponderance of values between .50 and .55 . Equation 6 was confirmed with $17 \mathrm{Ss}$, Eq. $4 \mathrm{a}$ with $7 \mathrm{Ss}$, Eq. 4b with $3 \mathrm{Ss}$, Eq. 5 a with $2 \mathrm{Ss}$, and Eq. $5 \mathrm{~b}$ with $1 \mathrm{~S}$. Consequently, most of the individual data reflected the findings for the groups, but not in an overwhelming fashion since data from almost half the Ss uphold either Eq. 4 or Eq. 5 .

Nonetheless, it seemed worthwhile to develop several models for the group data to represent the possible judgment strategies employed in the multidimensional situation.

Two new models were developed and tested with the data of Experiment 1. The first model was an extension of Eq. 1, based on the assumption that in the multidimensional case $\mathrm{Ss}$ estimate brightness and loudness components independently, and report their geometric mean. This geometric (G) model is represented by

$$
\mathrm{R}_{1,2}=\alpha_{1,2}\left[\left(\mathrm{~S}_{1}^{.52}\right)\left(\mathrm{S}_{2}^{.38}\right)\right]^{.5}
$$

or, since the normalized ratio value for $S_{1}$ equals the normalized ratio for $S_{2}$,

$$
\mathrm{R}_{1,2}=\alpha_{1,2} \mathrm{~S}_{1,2}^{.45} \text {, }
$$

where $n_{1,2}=.45=.5\left(n_{1}+n_{2}\right)$, but $n_{1,2}=\beta\left(n_{1}+n_{2}\right)$. Equation 7 then becomes

$$
\mathbf{R}_{1,2}=\alpha_{1,2}\left[\left(\mathbf{S}_{1}^{\mathrm{n}_{1}}\right)\left(\mathbf{S}_{2}^{\mathrm{n}_{2}}\right)\right]^{\beta},
$$

where $\beta$ now is a geometric averaging factor equal to .5, although its magnitude would vary somewhat for individual Ss. The exponent here is the exact value obtained for the first estimates (group data). An attempt was made to include multiplicative constants $(\alpha)$ from the equations for component attributes in Eq. 7, but the geometric mean of these values failed to yield the constant $(.80)$ obtained for the multidimensional condition (see Table 1). It is probably true that $\alpha_{1,2}$ is determined by factors unrelated to those responsible for the exponent.

The second model proposes a direct additive relation between estimates of the independent component dimensions. Specifically, it can be proposed that a linear relation exists between responses to the multidimensional stimuli and the sum of the responses to the component dimensions. Therefore,

$$
\mathbf{R}_{1,2}=\tau\left(\mathbf{S}_{1}^{\mathbf{n}_{1}}+\mathbf{S}_{2}^{\mathbf{n}_{2}}\right)+\gamma .
$$

For the first judgments in Experiment $1 \mathrm{Eq} .8$ predicts

$$
\mathrm{R}_{1,2}=\tau\left(\mathrm{S}_{1}^{.52}+\mathrm{S}_{2}^{.38}\right)+\gamma
$$




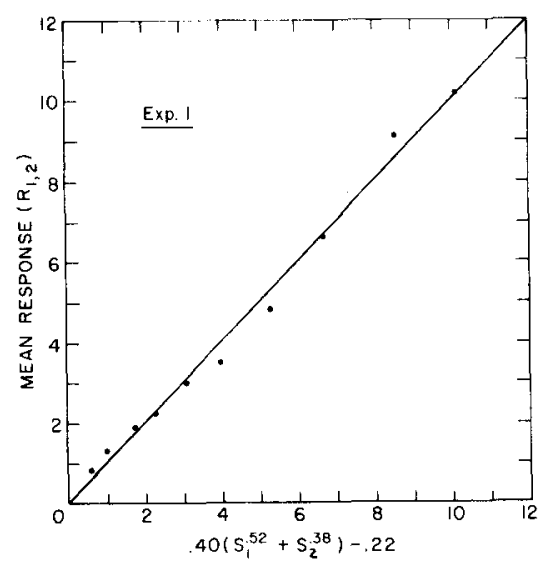

Fig. 2. Mean response magnitude $\left(R_{1,2}\right)$ for brightness-loudness pairs as a function of predictions from the additive model. Responses are geometric means calculated from 10 Ss' responses in Experiment 1. The slope (.40) and $y$-intercept $(-.22)$ of the theoretical equation were obtained by a least squares solution. The exponents (.52 and .38) were obtained from the data for loudness (.52) and brightness (.38) in Experiment 1 . The diagonal line represents a perfect match between theoretical and observed values. For more details, see the text.

The stimulus values used in Experiment 1 were substituted in Eq. 9 and a least squares solution performed to determine $\tau=.40$ and $\gamma=-.22$. The goodness of fit can be seen in Fig. 2, which compares the observed responses $\left(R_{1,2}\right)$ for first estimates with predicted responses based on Eq.9, including the empirically determined values for $\tau$ and $\gamma$. On the abscissa, we have the theoretical function (Eq. 9) for each of the stimulus magnitudes used in Experiment 1. The ordinate represents the empirical responses obtained in Experiment 1 for the multidimensional case. Both axes are linear, and consequently, a perfect fit of the additive model to the empirical results would be depicted by the diagonal line through the origin. The actual fit obtained is quite satisfactory.

\section{EXPERIMENT 2}

Except for the value of $\alpha_{1}$ (Eq. 7 of the geometric model), the geometric (G) and additive (A) models handled the data of Experiment 1 very well. In Experiment 2 the generality of these models was tested with a different arrangement of stimulus intensities.

This experiment was undertaken to test the generality of the $G$ and $A$ models developed to account for the results in Experiment 1. The same multidimensional attribute was used, but the paired intensity levels were different. Instead of correlated intensity values, an orthogonal design was employed, where each of five levels from the sound dimension was paired with each of five levels from the luminance dimension. It was hoped that this arrangement would provide a more stringent test of the $G$ and $A$ models.

\section{Method}

Subjects. The S population was the same as in Experiment 1. Twelve individuals participated in Experiment 2.

Apparatus. The apparatus and experimental rooms were essentially the same as in Experiment 1, but a few changes were introduced. The basic change was that the neutral density filters were mounted around the rim of a 12-in. Masonite disk which could be rotated automatically to interpose the correct filter in the path of the light beam.

The luminances used were $6.4,78.6$, $421.2,680.4$, and $1,808.9 \mathrm{fL}$, which represents a subset of the 10 intensities in Experiment 1. The sound intensities had the same normalized ratios as the luminances, in logarithmic form: $60,82,96,100.5$, and $109 \mathrm{~dB}$. A complete 5 by 5 orthogonal matrix was employed, yielding 25 brightness-loudness pairs.

Procedure. The method of magnitude estimation was used with the weakest intensity pair assigned the value of 1.0. Only estimates of the multidimensional stimuli were obtained. All other relevant procedural details were the same as in Experiment 1.

\section{Results and Discussion}

Arithmetic means were determined for each S's estimates of each stimulus, and the geometric mean of these values was used to summarize the group results. Due to difficulties encountered in developing a ratio scale of multidimensional stimuli whose components are orthogonally related, attempts to find an adequate solution were abandoned in favor of direct tests of the geometric and additive models. In order to test the applicability of the $\mathrm{G}$ model given in Eq. 7, the results of Experiment 1 were taken for $n_{1}, n_{2}$, and $\alpha_{1}, 2$. Then, with $S_{1}$ and $S_{2}$ taken from Experiment 2, an evaluation of the model was possible for each multidimensional pair. The results of this analysis are presented in Fig. 3, where $R_{1}$ is the geometric mean from the data in Experiment 2. As in Fig. 2, the abscissa represents the theoretical predictions of the model and the ordinate represents the

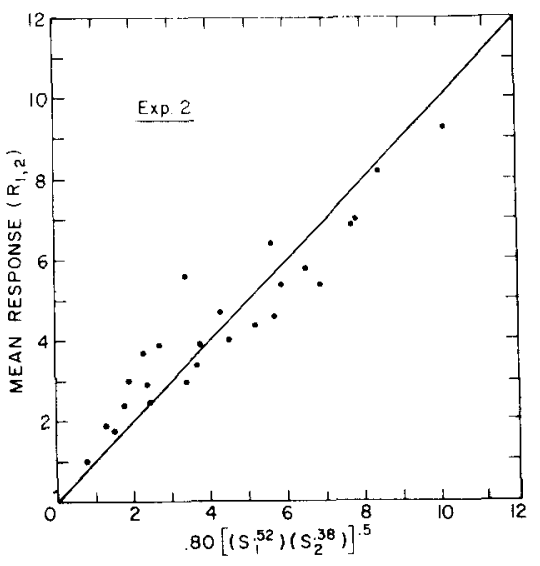

Fig. 3. Mean response magnitude $\left(R_{1,2}\right)$ for brightness-loudness pairs as a function of predictions from the geometric model. Each point is the geometric mean of responses obtained from $12 \mathrm{Ss}$ in Experiment 2. The multiplicative constant $(.80)$ and the exponents for loudness (.52) and brightness (.38) were obtained from the data of Experiment 1. For more details, see Fig. 2 and the text.

empirical group means. The solid diagonal line depicts a perfect match between hypothetical data and the predictions of the model. The fit of the $\mathbf{G}$ model is satisfactory, although some systematic deviations are evident: points in the lower half of the function are generally above the theoretical predictions, whereas points in the upper half are below the theoretical predictions.

The A model given in Eq. 8 was also evaluated. Here $n_{1}$ and $n_{2}$ were taken from Experiment 1, while $\tau$ and $\gamma$ were estimated from the data in Experiment 2 by least squares solution. The results are given in Fig. 4. The model handled these findings very well, and the scatter of points was less than comparable results for the $\mathrm{G}$ model (Fig. 3). On the other hand, a poor fit of the $A$ model was obtained when the actual $\tau$ and $\gamma$ values determined for Experiment 1 were used. The slope of the additive function for Experiment 1 was .40 , which is larger than the slope of .29 found for Experiment 2. Moreoever, the $y$-intercept $(\gamma)$ was positive in Experiment 2 and negative in Experiment 1. However, the smaller variability of points for the A model counteracts any advantage held by the $G$ model in regard to overall generality. The A model also suggests the existence of a much simpler and more plausible judgment process than does the $G$ model, but further empirical work will be required before a more definite decision can be made between the relative merits of the two models. Consequently, it does not 
seem very worthwhile at this stage to become involved in more elaborate statistical tests of possible differences between the models.

\section{OVERVIEW}

This study demonstrates that a direct scaling method (magnitude estimation) can be employed to obtain judgments of the intensity of a multidimensional stimulus. Additionally, we have shown that these judgments can be predicted with high confidence on the basis of judgments obtained for the unidimensional components. For the attributes of brightness and loudness, a power function offers an adequate description of results. Judgments of combined intensities from both attributes then can be predicted either by assuming that Ss take the geometric mean of their independent judgments of the two components (geometric model) or by assuming that Ss perform a linear averaging of their judgments of the components (additive model). Both models imply that $S s$ integrate the attributes of brightness and loudness at a cognitive level of processing rather than at a perceptual level involving the automatic summing of data from independent sensory channels. This lack of perceptual "integrality" can be defined operationally by the value of an averaging factor, $\beta$, found in the geometric model. The parameter $\beta$ represents the power to which judgments of the component dimensions are raised to produce judgments of the combined dimensions. When $\beta$ is 1.0 , the judgments of the components are summated, and it could be claimed

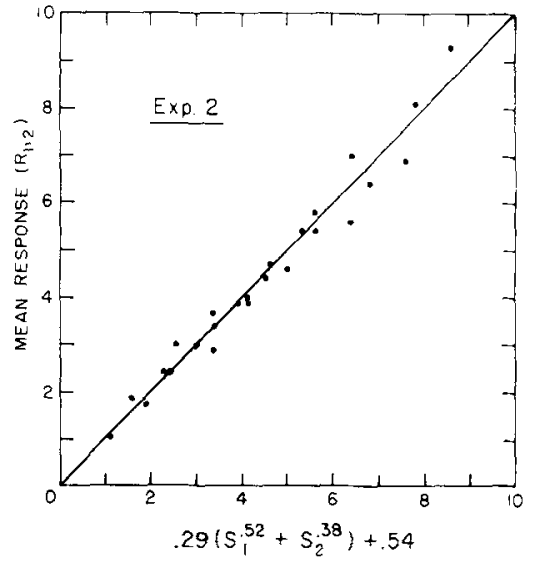

Fig. 4. Mean response magnitude $\left(R_{1}, 2\right)$, as a function of predictions from the additive model. Each point is the geometric mean of responses obtained from 12 Ss in Experiment 2. The exponents are based on data from Experiment 1 for loudness (.52) and brightness (.38), whereas the slope (.29) and $y$-intercept (.54) of the model were determined by a least squares solution. For more details, see Fig. 2 and the text.

that complete perceptual "integrality" is thereby achieved. In the present study, the group results yielded $\beta=.5$, which suggests that perceptual integrality was totally lacking. On the other hand, as $\beta$ continues on below .5 and approaches 0 , it could be claimed that perceptual "interference" between component dimensions becomes more pronounced. This possibility, as well as partial summation $(.5<\beta<1)$, was realized in the case of several individual Ss. The general usefulness of $\beta$ as a measure of perceptual "integrality" and "interference" for groups or individuals can only be assessed after more data have been collected with a wider variety of stimulus attributes.

\section{REFERENCES}

A N DER S ON N. H. Functional measurement and psychophysical judgment. Psychological Review, 1970 77, 153-170.

BAIRD, J. D. (Ed.) Human space perception: Proceedings of the Dartmouth conference. Psychonomic Monograph Supplements, 1970a, 3(13, Whole No. 45).

BAIRD, J. C. A cognitive theory of psychophysics: I. Information transmission, partitioning, and Weber's law. Scandinavian Journal of Psychology, $1970 \mathrm{~b}, 11,35-46$.

BAIRD, J. C. A cognitive theory of psychophysics: II. Fechner's law and Stevens' law. Scandinavian Journal of Psychology, 1970c, 11, 89-102.

BAIRD、J. C., ROMER, D., \& STEIN, T. Test of a cognitive theory of psychophysics: Size discrimination. Perceptual \& Motor Skills, 1970, 30, 495-501.

CURTIS, D. W., ATTNEAVE, F., \& HARRINGTON, T. C. A test of a two-stage model of magnitude judgment. Perception \& Psychophysics, 1968, 3, 25-31.

ERIKSEN, C. W. \& HAKE, H. W. $M$ ultidimensional stimulus differences and accuracy of discrimination, Journal of Experimental Psychology, 1955, 50, 153-160.

GARNER, W. R. Uncertainty and structure as psychological concepts. New York: Wiley, 1962 .

GARNER W. R. The stimulus in information processing. American Psychologist, 1970, 25, 350-358.

LOCKHEAD, G. R. Effects of dimensional redundancy on visual discrimination. Joumal of Experimental Psychology, 1966, 72, 95-104.

SHEPARD, R. N. Attention and the metric structure of the stimulus space. Journal of Mathematical Psychology, 1964, 1, 54-87.

(Accepted for publication May 12, 1971.) 